\title{
RADIO OBSERVATIONS OF MOLECULES IN NEARBY GALAXIES
}

\author{
J.B. Whiteoak \\ Division of Radiophysics, CSIRO, Sydney, Australia
}

The observation in 1963 of absorption due to the $18 \mathrm{~cm}$ groundstate transition of $\mathrm{OH}$ in the direction of Cas A (Weinreb et al., 1963) marked the first occasion on which a molecular cloud was detected at radio wavelengths. However, it was not until the later discovery of high-intensity OH emission (Weaver et al., 1965) that attention turned to nearby galaxies. Unfortunately, searches for $\mathrm{OH}$ emission in the Magellanic Clouds (McGee et al., 1965; Radhakrishnan, 1967) and in more distant galaxies (Roberts, 1967) were unsuccessful. The first detection of an $\mathrm{OH}$ transition, in absorption against the radio continuum of NGC 253 and 3034 (Weliachew, 1971), went almost unnoticed because the results were unconvincing. However, Whiteoak and Gardner (1973) and Nguyen-Q-Rieu et al. (1976) confirmed the existence of the absorption.

Transitions of other molecules abundant in our galaxy were later detected in nearby galaxies: the $6 \mathrm{~cm} 1_{11}-1_{10}$ transition of $\mathrm{H}_{2} \mathrm{CO}$ (Gardner and Whiteoak, 1974), the $2.6 \mathrm{~mm} \mathrm{~J}=1-0$ transition of CO (Rickard et al., 1975), the $3.2 \mathrm{~mm} \mathrm{~J}=1-0$ transition of HCN (Rickard et al., 1977b), and the $1.3 \mathrm{~cm} 616^{-5} 23$ transition of $\mathrm{H}_{2} \mathrm{O}$ (Churchwell et al., 1977.). Table I shows the molecules in chronological order of detection, for each of the 12 galaxies. Typical spectra and, for comparison, HI, $\mathrm{H} \alpha$, $\mathrm{H} 92 \alpha$, and $\mathrm{H} 166 \alpha$ profiles, are shown in Figures 1 to 3 . In addition to these results there has been a possible detection of the $9 \mathrm{~cm}$ $\mathrm{F}=1-0$ transition of $\mathrm{CH}$ in the Large Magellanic Cloud (Whiteoak and Gardner, 1978), while Rickard et al. (1977b) have reported a negative search for the $3.1 \mathrm{~mm} \mathrm{~J}=2-1$ transition of CS in NGC 253 and 3034 .

$\mathrm{OH}$. The main-line transitions (at 1665 and $1667 \mathrm{MHz}$ ) of the ${ }^{2} \pi_{3 / 2}$, $\vec{J}=3 / 2$ ground state of $\mathrm{OH}$ are best seen in absorption against the small-diameter (about 0'.5 arc) nuclear radio sources of dusty edge-on galaxies (Figs. 1a,b,c). Thus for NGC 253 and 4945 the observed peak line-to-continuum ratios at $1667 \mathrm{MHz}$ are about 0.03 and 0.1 , while for NGC 5236, an almost face-on spiral, the ratio is below 0.005 . The optical depths are uncertain because the absorption distribution over the continuum is not known. In NGC 4945, for an assumed optical depth of 0.3 (Whiteoak and Gardner, 1975) and a kinetic temperature of $10 \mathrm{~K}$, the total column density of $\mathrm{OH}$ is about $10^{17} \mathrm{~cm}^{-2}$. 
Table I

Molecules observed in external galaxies

\begin{tabular}{lll}
\hline \multicolumn{1}{c}{ Galaxy name } & Observed molecules & \multicolumn{2}{c}{ References } \\
\hline NGC 224 (M31) & $\mathrm{CO}$ & 2,12 \\
NGC 253 & $\mathrm{OH}, \mathrm{H}_{2} \mathrm{CO}, \mathrm{CO}, \mathrm{HCN}$ & $3,4,9,10,11,12,13,14$ \\
NGC 598 (M33) & $\mathrm{H}_{2} \mathrm{O}$ & 1,10 \\
NGC 1068 (M77) & $\mathrm{CO}$ & 10 \\
LMC & $\mathrm{CO}, \mathrm{H}_{2} \mathrm{CO}, \mathrm{OH}$ & $7,16,17$ \\
NGC 3031 (M81) & $\mathrm{CO}$ & 2 \\
NGC 3034 (M82) & $\mathrm{CO}, \mathrm{OH},(\mathrm{HCN})$ & $8,9,10,11,12,13$ \\
NGC 4945 & $\mathrm{OH}, \mathrm{H}_{2} \mathrm{CO}$ & $3,14,15$ \\
NGC 5055 (M63) & (CO) & 12 \\
NGC 5128 (Cen A) & $\mathrm{H} \mathrm{H}_{2} \mathrm{CO}, \mathrm{OH}$ & 5,6 \\
NGC 5194 (M51) & $\mathrm{CO}$ & $2,10,12$ \\
NGC 5236 (M83) & $\mathrm{CO}$ & 10 \\
\hline
\end{tabular}

1: Churchwe11 et al. (1977); 2: Combes et a1. (1977); 3,4,5,6: Gardner and Whiteoak (1974, 1975, 1976a,b); 7: Huggins et a1. (1975);

8: Nguyen-Q-Rieu et al. (1976); 9,10,11: Rickard et a1. (1975, 1977a,b); 12: Solomon and de Zafra (1975); 13: Weliachew (1971);

14,15,16,17: Whiteoak and Gardner (1973, 1975, 1976a,b).
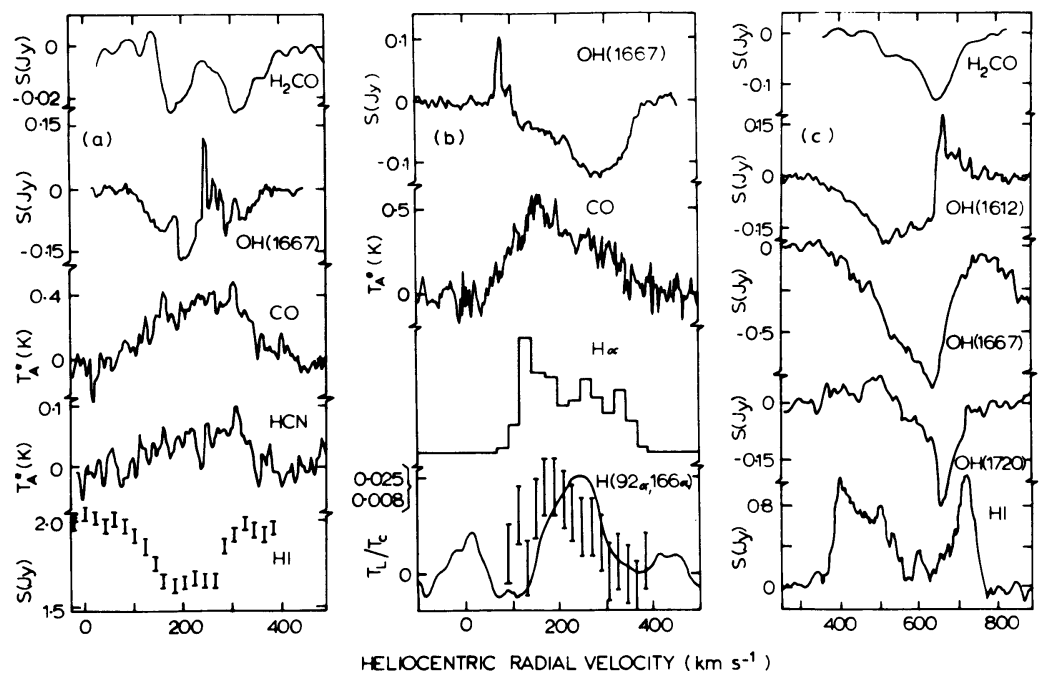

Fig. 1 - Line profiles towards the centres of the edge-on galaxies (a) NGC 253, (b) NGC 3034 (M82) and (c) NGC 4945. The vertical ordinates are flux density $(S)$, corrected antenna temperature $\left(T_{A}^{*}\right)$, or line-tocontinuum ratio $\left(\mathrm{T}_{\mathrm{L}} / \mathrm{T}_{\mathrm{C}}\right)$. In (b) the profile shown by error bars is for $\mathrm{H} 166 \alpha$; the appropriate scale is given by the lower value $(0.008)$. The $1665 \mathrm{MHz}$ profile has been excluded from (c) - it is similar in shape to the $1667 \mathrm{MHz}$ profile but reduced in amplitude by a factor of 1.8 . 
The absorption profiles for edge-on galaxies have velocity spreads centred approximately on the systemic velocities, and widths (of about $300 \mathrm{~km} \mathrm{~s}^{-1}$ ) not much less than the total variation in radial velocity associated with the overall rotation of each galaxy. For NGC 4945 these points are illustrated by comparison with the HI profile (Whiteoak and Gardner, 1976c), the width of which is determined by the overal1 rotation. However, the large widths of the absorption profiles must be the result of non-circular motions in the galaxies, because any widening due to differential galactic rotation across the extent of the radio continuum would be sma11 (as observed in the HI absorption observations of NGC 253 by Gottesman et al. (1976)). Moreover, because the radio continuum is believed to originate close to the centre of the galaxies, the non-circular motions must consist of motions both towards and away from the centre. Optically the latter have been observed in NGC 253 (Demoulin and Burbidge, 1970) and in NGC 3034 (Heckathorn, 1972).

The satellite $\mathrm{OH}$ ground-state transitions at 1612 and $1720 \mathrm{MHz}$ have been observed for NGC 253 (Gardner and Whiteoak, 1975) and NGC 4945 (Whiteoak and Gardner, 1975); in contrast to the main-line transitions they indicate systematic departures from local thermodynamic equilibrium. For both galaxies, instead of the profiles expected for LTE (fainter than the main-1ine profiles but similar in shape) there is a progressive change in the departures across the profiles, with enhanced $1612 . \mathrm{MHz}$ absorption and $1720 \mathrm{MHz}$ emission at the lower velocities, and the reverse situation at the higher velocities. Similar anomalies occur in the central regions of our galaxy (Whiteoak and Gardner, 1976d), and imply transfers in population between the $F=2$ and $F=1$ hyperfine levels of the OH ground state. Whiteoak and Gardner (1975) used the theory of Litvak (1969) to propose a model in which the transfers are caused by intense infrared radiation located in the nucleus of the galaxy. In this model the 'infalling' material is

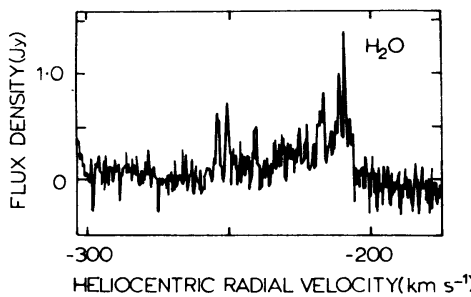

Fig. $3-\mathrm{H}_{2} \mathrm{O}$ spectrum for IC 133 in NGC 598. located near the nucleus while the outward motions take place further out.

In the $1667 \mathrm{MHz}$ profile for both NGC 253 and 3034 is a narrow ( 5 to $10 \mathrm{~km} \mathrm{~s}^{-1}$ ) feature which is probably maser emission (Whiteoak and Gardner, 1973). Although this emission is 1 to 2 orders of magnitude stronger than the most intense high-gain emission in our galaxy (the Class I OH emission sources associated with HII regions), it lacks the features of Class I emission. It is considerably weaker at 
$1665 \mathrm{MHz}$ and for NGC 253 at least is unpolarized, whereas Class I emission tends to be highly polarized and stronger at $1665 \mathrm{MHz}$. However, the features could result from low-gain amplification of the high brightness radiation in the galactic nuclei. The location may be settled when angular sizes of the emission become available.

In the LMC OH absorption is observed in the HII region N159 (Whiteoak and Gardner, 1976b). Despite intensive observations along the bar of this galaxy, no narrow-band emission above $0.15 \mathrm{Jy}$ in flux density has been detected. At this level, brighter Class I sources of our galaxy, if located at the distance of the LMC, would have been detectable.

$\underline{\mathrm{H}}_{2} \mathrm{CO}$. The $1_{10^{-1}}{ }_{11}$ transition is in absorption, and towards the nuclei of NGC 253 and 4945 the profiles are wide and generally similar in shape to the $\mathrm{OH}$ absorption, suggesting a common origin for the two molecules. However, the $\mathrm{H}_{2} \mathrm{CO}$ absorption is much fainter than the $\mathrm{OH}$ absorption, the maximum line-to-continuum ratios being only 0.02 and 0.08 for the two galaxies. The ratio of $\mathrm{H}_{2} \mathrm{CO}$ to $\mathrm{OH}$ absorption tends to increase with velocity as for the centre of our galaxy.

$\mathrm{H}_{2} \mathrm{CO}$ clouds well away from centres of galaxies have been detected in the LMC and NGC 5128. For the former, absorption was detected only towards N159 (Whiteoak and Gardner, 1976a); the profile is simple and comparatively narrow ( $\left.9 \mathrm{~km} \mathrm{~s}^{-1}\right)$, with a maximum line-to-continuum ratio (0.014) smal1 compared with the average (0.074) for our galaxy but comparable to values for some prominent regions (e.g. the Carina nebula). For NGC 5128, the profile towards the nucleus (Gardner and Whiteoak, 1976b) shows narrow ( 1.8 to $\left.3.5 \mathrm{~km} \mathrm{~s}^{-1}\right) \mathrm{H}_{2} \mathrm{CO}$ features, all with velocities exceeding the systemic velocity of $535 \mathrm{~km} \mathrm{~s}^{-1}$. The $\mathrm{H}_{2} \mathrm{CO}, \mathrm{OH}$ and $\mathrm{HI}$ absorption probably arises in several 'infalling' cold clouds located in the outer dust lane which overlies the nucleus. An additional cloud was detected in this lane and $4^{\prime}$ arc to the south-east of the nucleus; the absorption is probably against the extended continuum emission associated with the galaxy.

CO. Unlike $\mathrm{OH}$ and $\mathrm{H}_{2} \mathrm{CO}$, the $\mathrm{CO} \mathrm{J}=1-0$ transition appears in emission, and the chances of detection should be independent of galaxy inclination and background radio continuum. However, out of 32 bright galaxies observed, Rickard et a1. (1977a) detected CO emission in the nuclear regions of only 8 , where the galactic nuclei showed optical emission lines or contained radio continuum or strong infrared emission.

Figures 1(a),(b) contain the CO profiles observed towards the centre of NGC 253. (Rickard et al., 1977b) and NGC 3034 (Rickard et al., 1977a). Compared with the $\mathrm{OH}$ and $\mathrm{H}_{2} \mathrm{CO}$ absorption the $\mathrm{CO}$ spectra are broader, and the profile shapes, although possibly showing some similar large-scale structure, differ in detail. A difference is to be expected, because the $\mathrm{OH}$ absorption occurs in front of the small-diameter nucleus whereas the CO clouds are located anywhere along the lines of sight within a 65" arc beam. For NGC 3034 the CO profile resembles the Ha profile constructed by Rickard et al. (1977a) from the observations of Heckathorn (1972), suggesting a location for the CO in the dust of the optical filaments. The CO profile is also roughly similar to the H92 $\alpha$ and 
H166 profiles (Chaisson and Rodriguez, 1977; Shaver et al., 1977). The profile widths of NGC 5194 and 5236 are only about half those shown in the figures - probably because of the high inclination of the planes of these galaxies to the line of sight.

Maps of the CO distribution in NGC 253 and 3034 (Rickard et al., 1977a) show that the emission is highly concentrated towards the nuclei the half-intensity widths of the distributions were only $1 \frac{1}{2}$ to 2 times the 65" arc beamwidth. The distribution maximizes at a position on the major axis close to but displaced from the nucleus (by about $0^{\prime} .5$ arc) and has a velocity centroid that varies in position with a sense consistent with the general galactic rotation. These features, and the large profile widths (Solomon and de Zafra, 1975), are similar to those for the CO emission in our galaxy. Rickard et al. (1977a) have claimed a further similarity for the abundance ratios of $\mathrm{CO}$ to $\mathrm{OH}$ and $\mathrm{H}_{2} \mathrm{CO}$, although the calculations were based on some questionable assumptions.

CO emission has been detected in N159 in the LMC (Huggins et al., 1975), and along the dusty sides of spiral arms in NGC 224, 3031 and 5194 (Combes et al., 1977). The emission bandwidths are narrow (typically $20 \mathrm{~km} \mathrm{~s}^{-1}$ ) and the velocities are similar to $\mathrm{HI}, \mathrm{OH}$ and $\mathrm{H}_{2} \mathrm{CO}$ velocities in the same directions, although in NGC 224 there may be a slight systematic difference between the $\mathrm{CO}$ and HI velocities (F. Combes, private communication). Combes et al. (1977) have suggested that the CO cloud density is similar to that within the region 4 to $8 \mathrm{kpc}$ from the centre of our galaxy, but the claim was based on a sample of only seven CO detections.

HCN. The $J=1-0$ transition was detected in emission towards the centre of NGC 253 and possibly NGC 3034 (Rickard et al., 1977b). The profile for NGC 253 (Fig. 1a) is much fainter than the CO profile but similar in shape. Calculations based on an assumption (probably incorrect) of low optical depths yielded an abundance ratio of $\mathrm{HCN}$ to $\mathrm{CO}$ close to the value for our galaxy.

$\mathrm{H}_{2} \mathrm{O}$. Maser emission at $22 \mathrm{GHz}$ (Fig. 3) was detected in one HII region (IC 133) out of a total of 16 observed in NGC 598 by Churchwell et a1. (1977). The brightness temperature of the strongest line would be about $10 \%$ to $15 \%$ of that for W49 during its more quiescent periods, if W49 were at the distance of IC 133. However, IC 133 does not resemble the bright compact HII regions with which many of the $\mathrm{H}_{2} \mathrm{O}$ sources in our galaxy are associated.

This review has shown that a total of five molecules have been detected at radio wavelengths in 12 nearby galaxies. Most transitions have been observed towards the centres of dusty galaxies - the absorption profiles for edge-on galaxies provide unquestionable evidence for the existence of non-circular motions. There is little doubt that other molecules will be detected as better receiving equipment becomes available. 
Chaisson, E.J. and Rodriguez, L.F.: 1977, Astrophys. J. Lett. 214, L11.

Churchwe11, E., Witzel, A., Huchtmeier, W., Pauliny-Toth, I.,

Roland, J. and Sieber, W.: 1977, Astron. Astrophys. 54, 969.

Combes, F., Encrenaz, P.J., Lucas, R. and Weliachew, L.: 1977, Astron. Astrophys. 55, 311.

Demoulin, M.H. and Burbidge, E.M.: 1970, Astrophys. J. 159, 799.

Gardner, F.F. and Whiteoak, J.B.: 1974, Nature 247, 526.

Gardner, F.F. and Whiteoak, J.B.: 1975, Mon. Not. R. Astron. Soc. $173,77 \mathrm{P}$.

Gardner, F.F. and Whiteoak, J.B.: 1976a, Mon. Not. R. Astron. Soc. $175,9 \mathrm{P}$.

Gardner, F.F. and Whiteoak, J.B.: 1976b, Proc. Astron. Soc. Aust. 3,63 .

Gottesmann, S.T., Lucas, R., Weliachew, L. and Wright, M.C.: 1976, Astrophys. J. 204, 699.

Heckathorn, H.M.: 1972, Astrophys. J. 173, 501.

Huggins, P.J., Gillespie, A.R., Phillips, T.G., Gardner, F.F. and Knowles, S.H.: 1975, Mon. Not. R. Astron. Soc. 173, 69P.

Litvak, M.M.: 1969, Astrophys. J. 156, 471.

McGee, R.X., Robinson, B.J., Gardner, F.F. and Bolton, J.G.: 1965, Nature 208, 1193.

Nguyen-Q-Rieu, Mebold, U., Winnberg, A., Guibert, J. and Booth, R.: 1976, Astron. Astrophys. 52, 467.

Radhakrishnan, V.: 1967, Aust. J. Phys. 20, 203.

Rickard, L.J., Palmer, P., Morris, M., Zuckerman, B. and Turner, B.E.: 1975, Astrophys. J. Lett. 199, L75.

Rickard, L.J., Palmer, P., Morris, M., Turner, B.E. and Zuckerman, B.: 1977a, Astrophys. J. 213, 673.

Rickard, L.J., Palmer, P., Turner, B.E., Morris, M. and Zuckerman, B.: 1977b, Astrophys. J. 214, 390.

Roberts, M.S.: 1967, Astrophys. J. 148, 931.

Shaver, P.A., Churchwe11, E. and Rots, A.H.: 1977, Astron. Astrophys. 55,435 .

Solomon, P.M. and de Zafra, R.: 1975, Astrophys. J. Lett. 199, L79.

Weaver, H., Williams, D.R.W., Dieter, N.H. and Lum, W.T.: 1965, Nature 208, 29.

Weinreb, S., Barrett, A.H., Meeks, M.L. and Henry, J.C.: 1963, Nature 200, 829.

Weliachew, L.: 1971, Astrophys. J. Lett. 167, L47.

Whiteoak, J.B. and Gardner, F.F.: 1973, Astrophys. Lett. 15, 211.

Whiteoak, J.B. and Gardner, F.F.: 1975, Astrophys. J. Lett. 195, L81.

Whiteoak, J.B. and Gardner, F.F.: 1976a, Mon. Not. R. Astron. Soc. $174,51 P$.

Whiteoak, J.B. and Gardner, F.F.: 1976b, Mon. Not. R. Astron. Soc. $176,25 P$.

Whiteoak, J.B. and Gardner, F.F.: 1976c, Proc. Astron. Soc. Aust. 3, 71.

Whiteoak, J.B. and Gardner, F.F.: 1976d, Mon. Not. R. Astron. Soc. 174,627 .

Whiteoak, J.B. and Gardner, F.F.: 1978, submitted to Mon. Not. R. Astron. Soc. 


\section{DISCUSSION FOLLOWING PAPER II.5 GIVEN BY J.B. WHITEOAK}

WELIACHEW: A FIRST STEP TOWARD THE RADIAL DISTRIBUTION OF CO IN M31 Emission from carbon monoxide has been systematically searched along the major axis of $\mathrm{M} 31$, in the region of spiral arms where the first detection was done (Combes et al. 1977, A.A. 55, 331). A partial radial distribution of $\mathrm{CO}$ emission is derived showing that carbon monoxide is unambiguously located on the inner side of the HI spiral arms. This can be explained in the frame of the density wave theory of spiral structure. As we are inside the co-rotation radius molecular clouds are formed by the compression of the interstellar medium on the inner side of a spiral arm, assuming trailing arms. A11 Co and HI (Emerson 1976, M.N.R.A.S. 176, 321) velocities agree within the uncertainties in their determination.

BALDWIN: Your peak temperature seen in $\mathrm{CO}$ of $0.3 \mathrm{~K}$ occurs at a point where the projected density of $\mathrm{HI}$ is also at maximum. Comparison of the $\mathrm{CO}$ and $\mathrm{HI}$ at the individual points where the measurements were made might modify the conclusion about the displacement between $\mathrm{CO}$ and $\mathrm{HI}$.

EMERSON: With respect to the relative intensities of $\mathrm{CO}$ and $\mathrm{HI}$ in the $\mathrm{N}$ and $\mathrm{S}$ of $\mathrm{M} 31$, and the relative positions of $\mathrm{HI}$ and $\mathrm{CO}$ emission, I do not think that your comparison with Guibert's major axis HI distribution is valid. Your $\mathrm{CO}$ points in the $\mathrm{S}$ of $\mathrm{M} 31$ are not typical points in HI, being exceptionally bright "hot-spots", and are not represented adequately in a major axis HI cut. It is not meaningful to make a comparison of the intensities and positions of your individual co points observed with a $1^{\prime}$ beam, with the global radial distribution of HI deduced from Guibert's Nançay observations made with a beamwidth of $4^{\prime} \times 24^{\prime}$.

[see Chapter III for other papers on M31] 